\title{
Correction: A COVID-19 Pandemic Artificial Intelligence-Based System With Deep Learning Forecasting and Automatic Statistical Data Acquisition: Development and Implementation Study
}

Cheng-Sheng $\mathrm{Yu}^{1,2,3,4^{*}}, \mathrm{PhD}$; Shy-Shin Chang ${ }^{1,2^{*}}, \mathrm{MD}, \mathrm{PhD}$; Tzu-Hao Chang ${ }^{3,5}$, PhD; Jenny L Wu ${ }^{1,3}$, BSc; Yu-Jiun Lin $^{1,2}$, MD; Hsiung-Fei Chien ${ }^{6}, \mathrm{MD}, \mathrm{PhD}$; Ray-Jade $\mathrm{Chen}^{4,7,8}, \mathrm{MD}, \mathrm{MSc}$

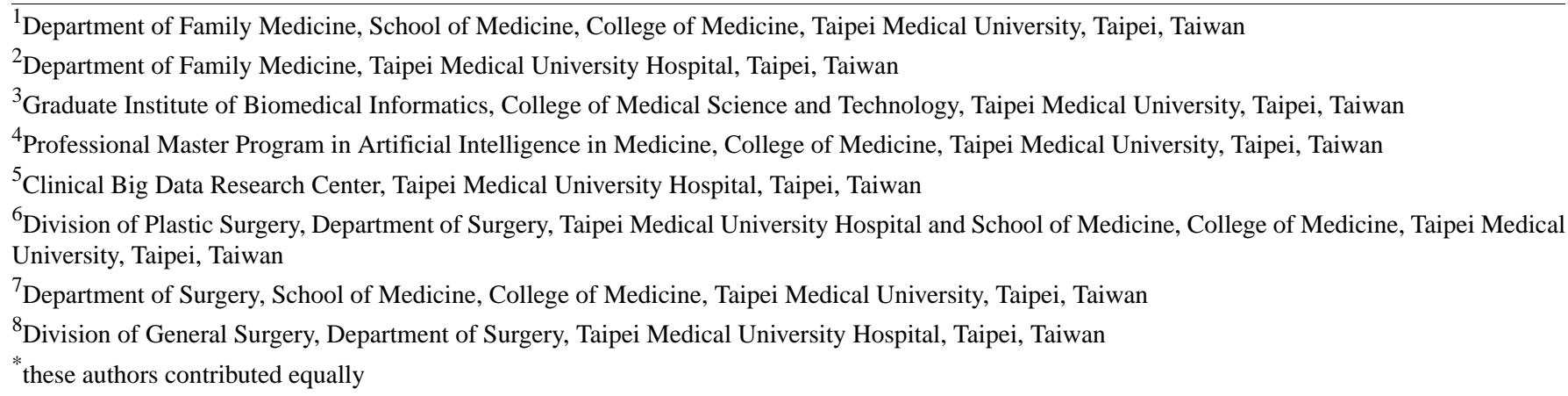

Corresponding Author:

Ray-Jade Chen, MD, MSc

Department of Surgery

School of Medicine, College of Medicine

Taipei Medical University

No.250, Wuxing St.,

Taipei, 11031

Taiwan

Phone: 886227372181 ext 3966

Email: rayjchen@tmu.edu.tw

\section{Related Article:}

Correction of: https://www.jmir.org/2021/5/e27806

(J Med Internet Res 2021;23(7):e31085) doi: 10.2196/31085

In "A COVID-19 Pandemic Artificial Intelligence-Based System With Deep Learning Forecasting and Automatic Statistical Data Acquisition: Development and Implementation Study" (J Med Internet Res 2021;23(5):e27806), the authors noticed two errors.

In the originally published manuscript, authors Cheng-Sheng $\mathrm{Yu}$ and Shy-Shin Chang should have been denoted as having contributed equally to the paper but were not. A footnote clarifying equal contribution has now been added to each of the aforementioned authors.

Additionally, in the originally published manuscript, the Acknowledgments section was omitted. In the corrected version, a new Acknowledgments section has been added with the following statement:
This study is supported by the Ministry of Science and Technology Grant (MOST 110-2314-B-038-025) and Higher Education Sprout Project by the Ministry of Education (MOE) in Taiwan (DP2-110-21121-01-A-09). No funding bodies had any role in study design, data collection and analysis, decision to publish, or preparation of the article.

The correction will appear in the online version of the paper on the JMIR Publications website on July 9, 2021, together with the publication of this correction notice. Because this was made after submission to PubMed, PubMed Central, and other full-text repositories, the corrected article has also been resubmitted to those repositories. 
This is a non-peer-reviewed article. Submitted 09.06.21; accepted 10.06.21; published 09.07.21.

Please cite as:

Yu CS, Chang SS, Chang TH, Wu JL, Lin YJ, Chien HF, Chen RJ

Correction: A COVID-19 Pandemic Artificial Intelligence-Based System With Deep Learning Forecasting and Automatic Statistical

Data Acquisition: Development and Implementation Study

J Med Internet Res 2021;23(7):e31085

URL: https://www.jmir.org/2021/7/e31085

doi: $10.2196 / 31085$

PMID: $\underline{34255678}$

(CCheng-Sheng Yu, Shy-Shin Chang, Tzu-Hao Chang, Jenny L Wu, Yu-Jiun Lin, Hsiung-Fei Chien, Ray-Jade Chen. Originally published in the Journal of Medical Internet Research (https://www.jmir.org), 09.07.2021. This is an open-access article distributed under the terms of the Creative Commons Attribution License (https://creativecommons.org/licenses/by/4.0/), which permits unrestricted use, distribution, and reproduction in any medium, provided the original work, first published in the Journal of Medical Internet Research, is properly cited. The complete bibliographic information, a link to the original publication on https://www.jmir.org/, as well as this copyright and license information must be included. 